\title{
Dynamics of EHD Laminar Plumes: scaling analysis and integral model
}

\author{
P.A. Vázquez ${ }^{1,2}$, A.T. Pérez ${ }^{2}$, A. Castellanos ${ }^{2}$ and P. Atten ${ }^{3}$ \\ ${ }^{1}$ Departamento de Física Aplicada, E.S.I., Avda. de los Descubrimientos, s/n, 41092 Sevilla, Spain, e-mail:pvazquez@cica.es, \\ 00-34-5-4486192, 00-34-5-4486003 (Fax) \\ ${ }^{2}$ Departamento de Electrónica y Electromagnetismo, Facultad de Física, Avda. Reina Mercedes s/n, 41012, Sevilla, Spain \\ ${ }^{3}$ Laboratoire d'Electrostatique et Materiaux diélectriques (C.N.R.S), Grenoble, France
}

\begin{abstract}
In this paper electrohydrodynamic (EHD) plumes are examined in the region far from the injecting electrode and the collector plate, for both two-dimensional and axisymmetric geometries. The relative importance of the conduction mechanisms (convection, drift and diffusion of electric charge) is analyzed. Diffusion turns out to be negligible compared to convection and drift for the experimental conditions. But the transverse drift (Coulomb repulsion) is of the same order of magnitude than convection. We find a set of three differential equations giving the evolution of the velocity at the center of the plume and the widths of the plume and the charged core inside.
\end{abstract}

\section{INTRODUCTION}

Injection of charge from an electrode into an insulating fluid defines a class of EHD flows. The Coulomb force, $\mathbf{f}=q \mathbf{E}$, that the electric field, $\mathbf{E}$, exerts on the space charge, $q$, induces a fluid motion which contributes to convect the charge. There is a drastic difference between gases and liquids. In gases the ion drift velocity is much higher than the gas flow induced by the Coulomb force, so the ions practically move along the electric field lines. The converse occurs in liquids where the typical fluid velocity is higher or much higher than the ion drift velocity ${ }^{1}$. The flow induced by the Coulomb force drastically affects the charge density distribution which in turn modifies the velocity. As a consequence there is a strong coupling between the charge density distribution and the velocity field.

We consider here the case of electrode geometries where the injection of charge arises from sharp electrodes. In these cases the contrast between gases and liquids is even more marked. In liquids the localized injection induces a flow structure similar to those of thermal plumes with a tendency to confine the charge. Several geometries can be considered, but in this paper we focus our attention on the two-dimensional and axisymmetric cases. The former arises when the sharp electrode is a blade or a wire, while the latter appears when the injecting electrode is a point or a needle. The resulting flow in the region far from the electrodes (we will call this zone the asymptotic region) is similar to that of thermal plumes arising from line or point sources of heat. Here only the case of laminar flow in liquids is examined.

Thermal plumes have been thoroughly studied ${ }^{2,3}$. The flow in the asymptotic region, for Prandtl numbers, $\operatorname{Pr} \gg 1$, $(\operatorname{Pr}=\nu / \kappa$ is the Prandtl number, $\nu$ the kinematic viscosity of the fluid and $\kappa$ the thermal diffusivity) has a double boundary layer structure, with an inner thermal layer, of width $\delta_{T}(x)$, and an outer hydrodynamic layer, of width $\delta_{l}(x)$, representing the volume of fluid put into motion ( $x$ is the coordinate along the flow main direction). These thermal plumes can be described in terms of self-similar solutions. The physical quantities of the plume scale with $x$, and the partial differential equations can be converted into a set of ordinary differential equations in a self-similar variable, $\eta=y / \delta_{l}(x)$, with $y$ the transverse coordinate.

Several attempts have been made to apply this technique to the EHD problem. The first study was carried out by Zhakin ${ }^{4}$. In his paper, the author neglects the contribution of Coulomb repulsion in the evolution of the charged zone, retaining the diffusive term. In this way, the problem turns out to be exactly analogous to the thermal one, with the charge density $q$ playing the role of temperature. Self-similar solutions for both two-dimensional and axisymmetric EHD plumes were given. Pérez et $\mathrm{al}^{5}$ showed that Coulomb repulsion is dominant compared to diffusion, at least in the usual experimental conditions. Vázquez et $\mathrm{al}^{6}$ analyzed both thermal and EHD plumes emphasazing their analogies and differences. They showed that the equations are similar to those of the thermal plumes in the limit $\operatorname{Pr} \rightarrow \infty$, when diffusion of charge, drift and Coulomb repulsion are neglected compared to charge convection. From a physical point of view, the width of the inner charged layer (the heated layer for the thermal plumes) is zero in this limit. Obviously, this is not a complete solution of the problem, for in that case both Coulomb repulsion and diffusion of charge would spread the charged region. Even more, for the two-dimensional geometry the velocity of the fluid in the plane of symmetry tends to a finite value for $\operatorname{Pr} \rightarrow \infty^{7,8}$, but for the axisymmetric case the velocity in the axis diverges as $\sqrt{\ln P r} 6$. What really happens in practical situations is that the plume enters the similarity zone with a charged region of finite width, $\delta_{q}$, due to the finite radius of the injecting electrode and to the effect of Coulomb repulsion in its vicinity ${ }^{9}$. To avoid this singularity, an effective Prandtl number was defined, $\operatorname{Pr} r^{e f}=\left(\delta_{l} / \delta_{q}\right)^{2}$, taking 
into account that the Prandtl number gives the relation between the widths of the thermal and hydrodynamic layer, $\operatorname{Pr}=\left(\delta_{l} / \delta_{T}\right)^{2}$.

Takashima et al. ${ }^{10}$ have done a numerical simulation for both two-dimensional and axisymmetric EHD plumes. They used finite differences to calculate the velocity field and the charge simulation method to determine the electric field. With the aim of reducing the computing time, they imposed a constant width of the charged layer, a rather drastic assumption not justified in the paper. In this way they obtained numerical values of the velocity and a relation between the potential difference and the current that they claim agree with their experiments. However, the underlying physical mechanisms are not clear in this numerical simulation, and the application of the calculations to other set of experimental data is not straightforward. An exact numerical solution of the whole problem is an extremely difficult task, because of the different length scales involved and the coupling between the electric field, the charge distribution and the velocity field.

Integral methods can give valuable insights in this difficult problem. McCluskey and Pérez ${ }^{11}$ used these methods for the first time. Imposing several hypotheses obtained from the experimental observation of EHD plumes, and neglecting diffusion of charge and Coulomb repulsion, they obtained expressions for the velocity in the symmetry plane of the plume and the width of the hydrodynamic layer in the asymptotic region. It must be underlined that these expressions are similar to those obtained by Zhakin. An order of magnitude analysis has been performed by Malraison et al. ${ }^{12}$ for axisymmetric and turbulent plumes following the same approach.

In the two-dimensional case, and for weak currents, the effect of space charge is negligible in comparison with convection under typical experimental conditions. In the axisymmetric case this is not true. Atten et al. ${ }^{13}$ considered the problem of EHD axisymmetric plumes including the effect of Coulomb repulsion with the use of integral methods. Making several hypotheses about the velocity profile, they obtained a set of differential equations for the velocity on the axis and the widths of the charged and hydrodynamic layers. However, their analysis is not complete and the results depend on an extra assumption concerning the ratio of the charged and hydrodynamic layers.

In this paper, we focus our attention in the behavior of laminar EHD plumes, for both 2D and axisymmetric geometries. We discuss in detail the relative importance of the three mechanisms of transport of electric charge in plumes: diffusion, drift (including Coulomb repulsion) and convection. It is shown that in the charged core and along the transverse coordinate, Coulomb repulsion is of the same order of magnitude than charge convection and cannot be neglected. The inclusion of Coulomb repulsion in the equations prevents them from having self-similar solutions. We extend the integral methods, used by Atten et al., including now the global conservation of energy. In this way, a set of differential equations is found for the velocity at the center of the plumes and the widths of the charged and hydrodynamic layers. In these equations the explicit dependence of the longitudinal electric field with $x$ appears. Therefore, we have to provide a function $E(x)$, along with the corresponding initial conditions. We have solved numerically the obtained equations for an imposed electric field and different sets of initial conditions. Similar values are obtained for the magnitudes involved in all cases.

\section{FORMULATION OF THE PROBLEM}

\section{A. Hydrodynamic equations}

We consider the steady electrohydrodynamic flow occurring between a blade or a needle and a plate a distance $d$ apart. In the first case, the structure of the flow is $2 \mathrm{D}$, and it is axisymmetric in the second case. The flow takes the form of a plume, originated at the point or the blade, that impinges upon the plate. The velocity in the zone of recirculation is much smaller than in the plume. For this reason one can analyze the motion considering a plume-like flow in a non-moving ambient liquid. This overall structure is sketched in figure 1.

We examine the problem in the asymptotic region, that is, far from both the injector and the plate. In this asymptotic region the flow has a double boundary-layer structure, with an inner charged core of typical scale $\delta_{q}$, and an outer hydrodynamic layer, of typical scale $\delta_{l}$. This layer represents the volume of liquid put into motion by the electric forces. We have $\delta_{q}, \delta_{l} \ll d$. The usual approximations in boundary-layer analysis are applicable here. For the Navier-Stoke equation the transverse derivatives are much higher than longitudinal derivatives, and the ambient pressure, $p_{0}(x)$, is impressed in the boundary layer (in our case $p_{0}$ is independent of $x$, as a consequence of neglecting recirculation, and therefore the pressure gradient is negligible). As a consequence the mechanical equations reduce to

$$
\begin{gathered}
\frac{\partial}{\partial x}\left(y^{k} u\right)+\frac{\partial}{\partial y}\left(y^{k} v\right)=0 \\
u \frac{\partial u}{\partial x}+v \frac{\partial u}{\partial y}=\frac{\nu}{y^{k}} \frac{\partial}{\partial y}\left(y^{k} \frac{\partial u}{\partial y}\right)+\frac{q E_{x}}{\rho}
\end{gathered}
$$


with $k=0$ for the $2 \mathrm{D}$ case and $k=1$ for the axisymmetric one. Here, $x$ and $y$ are the longitudinal and transverse coordinates (in the case of axisymmetric plumes $y$ is the radial coordinate), $u$ and $v$ are the longitudinal and transverse components of the liquid velocity, $q$ is the density of charge, $E_{x}$ is the longitudinal electric field, and $\rho$ and $\nu$ are the density and the kinematic viscosity of the liquid, respectively. As usual in boundary layer analysis, the conservation equation of the transverse component of momentum can be ignored ${ }^{14}$.

Following the usual analysis for thermal plumes ${ }^{2}$, we may derive estimates for the longitudinal velocity, $U$, and $\delta_{l}$ by stating that the inertial, viscous and force terms are of the same order

$$
U \sim\left(\frac{q E_{x} x}{\rho}\right)^{1 / 2}, \quad \frac{\delta_{l}}{x} \sim\left(\frac{q E_{x} x^{3}}{\rho \nu^{2}}\right)^{-1 / 4} .
$$

In analogy with the Grashof number defined for thermal plumes, we can define the electric Grashof number, Grel $=$ $(U x / \nu)^{2}=\left(q E_{x} x^{3} / \rho \nu^{2}\right)$, which represents the ratio of electric and viscous forces. These expression are of formal rather than practical interest because $q$ is not known a priori. In the following we turn toward more satisfactory expressions.

\section{B. Electric equations}

Two equations describe the electric aspects of the problem: the Poisson equation and the conservation of charge, in steady conditions,

$$
\begin{aligned}
& \nabla \cdot \mathbf{E}=\frac{q}{\epsilon}, \\
& \nabla \cdot \mathbf{j}=0 .
\end{aligned}
$$

Here $\epsilon$ is the permittivity and $\mathbf{j}$ the current density. Dielectric liquids of very poor conductivity are not ohmic. Space charge may appear in these liquids under certain restrictions ${ }^{1}$. Essentially, the space charge is observable when the transit time of ions between electrodes $d^{2} / K V$ ( $K$ being the ionic mobility and $V$ the applied voltage) is smaller than the space charge relaxation time $\epsilon / \sigma$ ( $\sigma$ being the conductivity). Under this condition, the density current has three components: the drift of ions with respect to the liquid, $K q \mathbf{E}$, the charge convection, $q \mathbf{u}$ and the charge diffusion $-D \nabla q$. Thus, $\mathbf{j}=q(K \mathbf{E}+\mathbf{u})-D \nabla q$, and eq. (3b) writes, in $2 \mathrm{D}$ geometry,

$$
\begin{array}{r}
K E_{x} \frac{\partial q}{\partial x}+K E_{y} \frac{\partial q}{\partial y}+\frac{K q^{2}}{\epsilon}+u \frac{\partial q}{\partial x}+v \frac{\partial q}{\partial y}- \\
-D \frac{\partial^{2} q}{\partial x^{2}}-D \frac{\partial^{2} q}{\partial y^{2}}=0
\end{array}
$$

The first term is related to the longitudinal electric drift, the second and third terms are due to the field created by the space charge (the Coulomb repulsion), the fourth and fifth terms are related to the convection of electric charge due to the liquid motion and the last two terms are due to charge diffusion.

\section{Basic scales}

From the structure of EHD plumes a more satisfactory set of estimates can be obtained. If the electric charge remains confined into the plume the current is (current per unit length for 2D plumes)

$$
\begin{aligned}
& J \approx \bar{q} 2 \delta_{q}\left(U+K \bar{E}_{x}\right) \text { two-dimensional case } \\
& I \approx \bar{q} \pi \delta_{q}^{2}\left(U+K \bar{E}_{x}\right) \text { axisymmetric case }
\end{aligned}
$$

with $\bar{q}, U$ and $\bar{E}_{x} \sim V / d$ the typical scales of the density of charge, the axial velocity and the axial electric field. From now on we assume $\delta_{q}$ to be smaller enough than $\delta_{l}$ for the previous expressions to be valid. In most EHD flows, including plumes, the ion drift velocity is negligible compared to the liquid velocity ${ }^{15}, K \bar{E}_{x} \ll U$. Now, integrating (1b) across an $x$-constant plane, and stating that the inertial, viscous and electric terms are of the same order we obtain 


$$
\begin{aligned}
U & \sim\left(J^{2} \bar{E}_{x}^{2} x / \rho^{2} \nu\right)^{1 / 5} \\
\delta_{l} & \sim\left(\rho \nu^{3} x^{2} / J \bar{E}_{x}\right)^{1 / 5} \text { two-dimensional case } \\
& U \sim\left(I \bar{E}_{x} / \rho \nu\right)^{1 / 2} \\
\delta_{l} & \sim\left(\rho \nu^{3} x^{2} / I \bar{E}_{x}\right)^{1 / 4} \quad \text { axisymmetric case }
\end{aligned}
$$

For 2D plumes the typical experimental values ${ }^{5}\left(J=10^{-8}-10^{-7} \mathrm{~A} \mathrm{~m}^{-1}, \overline{\mathrm{E}}_{\mathrm{x}}=10^{6} \mathrm{~V} \mathrm{~m}^{-1}, \rho=10^{3} \mathrm{Kg} \mathrm{m}^{-} 3, \nu=\right.$ $\left.2 \times 10^{-5} \mathrm{~m}^{2} \mathrm{~s}^{-1}, \mathrm{x} \sim 10^{-2} \mathrm{~m}\right)$ give $u \sim 3-9 \mathrm{~cm} / \mathrm{s}, \delta_{1} \sim 1.5-2.4 \mathrm{~mm}$. For axisymmetric plumes ${ }^{12}\left(I=10^{-8}\right)$ we get $u \sim 1 \mathrm{~m} / \mathrm{s}, \delta_{1} \sim 300-530 \mu \mathrm{m}$. In the following, we will use these values for the estimates.

\section{Charge transport mechanisms}

We now discuss the relative importance, in both geometries, of the three mechanisms of transport of charge in equation(4), that is, diffusion, electric drift and convection by the liquid.

\section{Diffusion vs. drift}

Longitudinal and transverse diffusion must be considered separately, as the length scales are quite different. In the asymptotic region the longitudinal scale of change of the density of charge is $d$, and the longitudinal electric field scale is $E_{x} \sim V / d$. Comparing the terms of (4) corresponding to diffusion and drift in the $x$ direction we have

$$
\frac{D \partial^{2} q / \partial x^{2}}{K E_{x} \partial q / \partial x} \sim \frac{D}{K V}=\frac{\kappa_{B} T}{e V},
$$

with $\kappa_{B}$ the Boltzmann constant, $T$ the absolute temperature and $e$ the electric charge of the ions. We have used the Einstein relation, $D / K=k_{B} T / e$, to derive (7). In usual experimental conditions, $(T=300 \mathrm{~K}, \mathrm{~V}=10 \mathrm{kV}, e$ the electron charge) the value of $(7)$ is $2.5 \times 10^{-6} \ll 1$. This shows that longitudinal diffusion is fully negligible compared to drift.

The transverse length scale for the charge density change is $\delta_{q}$, the typical width of the charged core. The transverse electric field mainly arises from the spatial charge density. In order to estimate it, we model this charge density as an infinite layer of width $2 \delta_{q}$ and the typical uniform density of charge $\bar{q}$, in the 2D case, and as an infinite cylinder of radius $\delta_{q}$ in the axisymmetric case. Using the Gauss law we obtain $E_{y} \sim \bar{q} \delta_{q} / \epsilon$. From (5) the transverse electric field is

$$
E_{y} \sim\left\{\begin{array}{cl}
J / 2 \epsilon U & \text { two-dimensional, } \\
I / \pi \epsilon U \delta_{q} & \text { axisymmetric. }
\end{array}\right.
$$

Comparing the transverse drift and diffusion in (4) we have

$$
\frac{D \partial^{2} \bar{q} / \partial y^{2}}{K E_{y} \partial \bar{q} / \partial y} \sim\left\{\begin{array}{cl}
2 \epsilon U k_{B} T / e J \delta_{q} & \text { two-dimensional } \\
\pi \epsilon U k_{B} T / e I & \text { axisymmetric. }
\end{array}\right.
$$

From the above estimates

$$
\frac{D \partial \bar{q} / \partial y}{\bar{q} K E_{y}} \sim \begin{cases}10^{-6} / \delta_{q} & \text { two-dimensional } \\ 3 \times 10^{-5} & \text { axisymmetric. }\end{cases}
$$

The conclusion is that diffusion effects are negligible compared to drift if $\delta_{q}$ is greater than a few $\mu \mathrm{m}$ in the $2 \mathrm{D}$ case, and for any value of $\delta_{q}$ in the axisymmetric case. Hence, diffusion will be neglected in the following derivation.

Remark that, if there would be other physical situations for which, in the 2D case, charge diffusion would be dominant over drift the problem would be completely analogous to the thermal case. This situation was considered by Zhakin ${ }^{4}$. The electric Schmidt number, $S c^{e l}=\nu / D$, then plays the role of the $\operatorname{Pr}$ number. Since $S c^{e l}=\nu / D \sim$ $10^{5}-10^{6}$, we could apply the self-similar solutions found elsewhere ${ }^{5,6}$. 
Consider now the relative importance of drift and convection terms in (4) at the boundary of the charged region. Taking $y \sim \delta_{q}$ as transverse length scale, from the continuity eq. (1a) the scale of transverse velocity is $v \sim\left(\delta_{q} / x\right) U$. For the electric field we deduced in II D 1, $E_{x} \sim V / d, E_{y} \sim \bar{q} \delta_{q} / \epsilon$. Thus, the order of magnitude of the different terms is $K \bar{q} V / x d$ for the longitudinal drift, $K \bar{q}^{2} / \epsilon$ for the Coulomb repulsion and $U \bar{q} / x$ for the convection.

Comparing the longitudinal terms due to drift and convection we get

$$
\frac{K E_{x}(\partial q / \partial x)}{u(\partial q / \partial x)} \sim \frac{K V / d}{U} \ll 1,
$$

in the usual experimental conditions. So longitudinal drift can be fully neglected.

For the transverse components we have

$$
\frac{K E_{y}(\partial q / \partial y)}{u(\partial q / \partial x)} \sim \frac{K x \bar{q}}{\epsilon U} .
$$

An estimation of $\bar{q}$ is needed. The density of charge can be expressed, in the absence of diffusion, in terms of the time during which Coulomb repulsion acts, i.e. the time required for the ions to go from the injector to the point of coordinates $(x, y)$. Neglecting charge diffusion, the charge conservation equation in steady conditions can be written ${ }^{1}$ :

$$
(K \mathbf{E}+\mathbf{u}) \cdot \nabla q=-\frac{K q^{2}}{\epsilon} \Rightarrow \frac{d q}{d t}=-\frac{K q^{2}}{\epsilon},
$$

Here $\mathrm{d} / \mathrm{dt}$ is a total derivative associated to the resulting velocity $(K \mathbf{E}+\mathbf{u})$ of charge carriers. Eq. (13) is readily integrated to obtain

$$
q(t)=\frac{q_{i}}{1+t / \tau_{r}}
$$

Here, $q_{i}$ is the density of charge at the injector, $\tau_{r}=\epsilon / K q_{i}$ the typical evolution time. If $x \gg \delta_{q}$, we have in the core $t(x, y) \sim t(x, 0) \sim x / U$, because $K E_{x} \ll U$. In this way, $\bar{q}$ can be taken as the density of charge at the center of the plume, $\bar{q}(x)=q(x, y=0)=q_{0}(x)$. We define $\Omega(x)$ as the quotient of the two characteristic times

$$
\Omega=t(x) / \tau_{r} \sim K q_{i} x / \epsilon U
$$

If $\Omega \ll 1$, (14) gives $q_{0}(x) \approx q_{i}$ and $(12)$ is very small. In this case, Coulomb repulsion is negligible in the spreading of the charged region and (4) writes

$$
u \frac{\partial q}{\partial x}+v \frac{\partial q}{\partial y}=0
$$

The problem is formally analogous to that of thermal plumes in the limit $\operatorname{Pr} \rightarrow \infty$ and self-similar solution are conceivable.

If $\Omega \gg 1, q_{0}(x)$ takes the asymptotic value $q_{0}(x) \sim \epsilon / K t \sim \epsilon U / K x$. Therefore, (12) is of order 1 , and Coulomb repulsion and convection are of the same order. In this case (4) writes

$$
\frac{K q^{2}}{\epsilon}+u \frac{\partial q}{\partial x}+\left(K E_{y}+v\right) \frac{\partial q}{\partial y}=0 .
$$

Eqs. (16) or (17), with (1a)-(1b), define the dynamics of EHD plumes. We must now estimate the value $\Omega$ in experimental conditions.

\section{Estimation of $\Omega$}

The crucial parameter is the initial density of charge, $q_{i}$. It can be related to the current because, at the electrodes, the liquid velocity vanishes, and conduction is due only to drift. We consider first the $2 \mathrm{D}$ case.

Modeling the electrode as an hyperbole, and in the absence of space charge, the electric field can be calculated by means of conformal mapping ${ }^{16}$. It gives $E_{e l}=\sqrt{2} V / \pi d^{1 / 2} r_{0}^{1 / 2}, r_{0}$ being the radius of curvature of the electrode. 
The injection of charge occurs when $E_{e l}$ reaches a threshold value, $E_{s}$. The space charge modifies the electric field on the injector and, similarly to what occurs for corona effect in gases ${ }^{17}$, for $V>V_{S}$ the steady electric field in the vicinity of the needle tip keeps nearly constant values (corresponding to the harmonic field for $V=V_{s}$ ) independently of the current. From experiments in mineral and silicone oils ${ }^{5}$ we get $E_{s} \sim 0.1 \mathrm{MV} \mathrm{cm}^{-1}$. In order to get the order of magnitude of the injected charge we supposed the blade to inject into an angle $0<\theta<\theta_{0}$ (see figure 2). In the vicinity of the surface of the point, the liquid must be at rest, and only the drift can transport the electric charge. For $\theta_{0}=\pi / 2$ the current per unit length is $J=q_{i} \pi r_{0} K E_{s}$. Taking $r_{0}=5 \mu \mathrm{m}$, and $K=10^{-9} \mathrm{~m}^{2} \mathrm{~V}^{-1} \mathrm{~s}^{-1}$ one obtains

$$
q_{i} \sim \frac{J}{\pi r_{0} K E_{s}} \sim \begin{cases}6 \times 10^{-2} \mathrm{Cm}^{-3} & J=10^{-8} \mathrm{~A} \mathrm{~m}^{-1}, \\ 6 \times 10^{-1} \mathrm{C} \mathrm{m}^{-3} & J=10^{-7} \mathrm{~A} \mathrm{~m}^{-1} .\end{cases}
$$

Similar considerations can be made for axisymmetric plumes. The harmonic field is calculated approximating the needle by an hyperboloid( Laplace equation between an hyperboloid and a plate ${ }^{16,18}$ ). The field at the tip of the needle is $E_{e l}=2 V /\left(r_{0} \ln \left(4 d / r_{0}\right)\right)$. From experiments in mineral oils $\mathrm{s}^{13}, E_{s} \sim 4 \mathrm{MV} \mathrm{cm}^{-1}$. This value of $E_{s}$ is similar to that obtained from experiments in cyclohexane and liquid argon ${ }^{19,20}$. In order to get the order of magnitude of $q_{i}$, we simplify further the geometry modeling the point by a hemisphere of radius $r_{0}$. Taking $\theta_{0}=\pi / 2$, the current is $I \sim 2 \pi r_{0}^{2} K E_{s} q_{i}$. We get ${ }^{13}\left(K=10^{-9} \mathrm{~m}^{2} \mathrm{~V}^{-1} \mathrm{~s}^{-1}\right)$

$$
q_{i} \sim \frac{I}{2 \pi r_{0}^{2} K E_{s}} \sim\left\{\begin{array}{cl}
160 \mathrm{C} \mathrm{m}^{-3} & I=10^{-8} \mathrm{~A}, \\
1600 \mathrm{C} \mathrm{m}^{-3} & I=10^{-7} \mathrm{~A} .
\end{array}\right.
$$

From (19) and (18) the value of the parameter $\Omega$ can be estimated for both geometries, planar $\left(\Omega_{p}\right)$ and axisymmetric $\left(\Omega_{t}\right)$. Taking the typical values estimated in II C we get, for $x=1 \mathrm{~cm}$ and $\epsilon_{r}=2$,

$$
\Omega_{p} \sim\left\{\begin{array} { c c } 
{ 0 . 3 } & { J = 1 0 ^ { - 8 } \mathrm { A } \mathrm { m } ^ { - 1 } , } \\
{ 3 } & { J = 1 0 ^ { - 7 } \mathrm { A } \mathrm { m } ^ { - 1 } . }
\end{array} \quad \Omega _ { t } \sim \left\{\begin{array}{cc}
90 & I=10^{-8} \mathrm{~A}, \\
900 & I=10^{-7} \mathrm{~A} .
\end{array}\right.\right.
$$

We see that only in the 2D case, and for low values of the intensity of the current, the effect of Coulomb repulsion can be neglected.

\section{E. Boundary conditions}

The boundary conditions are given by the geometry of the problem. At the center line of the plumes, the distributions of longitudinal velocity and charge have a maximum and the transverse velocity must vanish. On the other hand, the longitudinal velocity far from the plume vanishes. We have, for both geometries,

$$
\begin{gathered}
\left.\frac{\partial u}{\partial y}\right|_{y=0}=\left.\frac{\partial q}{\partial y}\right|_{y=0}=\left.v\right|_{y=0}=0 \\
\left.u\right|_{y \rightarrow \infty}=0
\end{gathered}
$$

At steady state, the intensity of current per unit length $J$, in two-dimensional plumes, and the intensity current $I$ in axisymmetric plumes, must be a constant at any plane normal to the axis of the plume. Using again that $K E_{x} \ll U$,

$$
\begin{gathered}
J=\int_{-\infty}^{+\infty} q u d y \\
I=2 \pi \int_{0}^{+\infty} q u y d y
\end{gathered}
$$




\section{INTEGRAL ASYMPTOTIC SOLUTIONS}

When Coulomb repulsion is negligible, self-similar solutions to (1a)-(1b) in both geometries have been found in the case of constant electric field, the charge remaining confined into an infinitely thin layer or cylinder, so that $\delta_{q}$ is taken to be null ${ }^{6}$. In the $2 \mathrm{D}$ case we obtain

$$
\begin{gathered}
u(x, y)=\left(\frac{4 J^{2} E^{2}}{\rho^{2} \nu}\right)^{1 / 5} x^{1 / 5} f^{\prime}(\eta) \\
\delta_{l}(x)=\left(\frac{16 \rho \nu^{3}}{J E}\right)^{1 / 5} x^{2 / 5} .
\end{gathered}
$$

Here $\eta=y / \delta_{l}(x)$ is the self-similar variable and $f(\eta)$ is the self-similar stream function. In the axisymmetric case

$$
\begin{gathered}
u(x, y)=\left(\frac{I E}{2 \pi \rho \nu}\right)^{1 / 2} \frac{f^{\prime}}{\eta}, \\
\delta_{l}(x)=\left(\frac{2 \pi \rho \nu^{3}}{I E}\right)^{1 / 4} x^{1 / 2} .
\end{gathered}
$$

These solutions cannot describe the internal structure of the charged core. Even more, in the axisymmetric case, in the limit $\delta_{q} \rightarrow 0$, the velocity tends to infinity ${ }^{6}$. The effect of Coulomb repulsion must be considered to fully understand the EHD plumes structure.

Self-similar solutions are no longer possible when the Coulomb repulsion term in (17) is not neglected. Now we present an integral method analysis, generalizing the previous works by McCluskey and Pérez ${ }^{11}$ and Atten et al. ${ }^{13}$, that can describe the behavior of the principal magnitudes of EHD plumes.

\section{A. 2D plumes}

If $\delta_{q}<\delta_{l}$, we can define an average velocity $u_{m}(x)$ so that

$$
J=\int_{-\infty}^{+\infty} q u d y=Q_{p}(x) u_{m}(x)
$$

with $Q_{p}(x)=\int_{-\infty}^{\infty} q d y$ the charge per unit length. So $u_{m}$ is the average value of the axial velocity of the liquid into the charged core. When $\delta_{q} \ll \delta_{l}, u_{m}$ coincides with the velocity at the center of the plume. Our aim is to find the equations relating $\delta_{q}, \delta_{l}$ and $u_{m}$.

Integrating (1b), with $k=0$, with respect to $y$ from $y=-\infty$ to $y=\infty$, using (1a) and (21a), after some manipulations, leads to

$$
\int_{-\infty}^{\infty} \frac{\partial u^{2}}{\partial x} d y=\int_{-\infty}^{\infty} \frac{q E_{x}}{\rho} d y
$$

If $\delta_{q}$ is small enough, the variations of the longitudinal electric field as a function of the coordinate $y$ can be neglected so that, to a first approximation, $E_{x}(y, x)=E_{0}(x)$ for $|y| \leq \delta_{q}$. Then, the right-hand side term in (26) can be expressed in terms of $Q_{p}(x)$. Multiplying $(26)$ by $u_{m}$ we get

$$
u_{m} \frac{d}{d x} \int_{-\infty}^{\infty} u^{2} d y=\frac{J E_{0}}{\rho} .
$$

and expresses that the variation of total momentum in a plane transverse to the flow equates the total force per unit length exerted by the imposed electric field on the liquid. Note that the viscous term does not contribute to the balance because it is an internal force.

Conservation of kinetic energy allows us to take the viscous effects into account. Multiplying (1b) by $u$ and manipulating it in the same way we obtain 


$$
\frac{d}{d x} \int_{-\infty}^{\infty} \frac{1}{2} u^{3} d y=-\nu \int_{-\infty}^{\infty}\left(\frac{\partial u}{\partial y}\right)^{2} d y+\frac{J E_{0}}{\rho}
$$

This equation expresses the balance between the kinetic energy of the liquid, the viscous losses and the power injected by the electric force. Finally, let us reconsider equation (25),

$$
J=Q u_{m} \approx 2 \bar{q}(x) \delta_{q}(x) u_{m}(x) \Rightarrow \delta_{q} u_{m} \approx \frac{J}{2 \bar{q}(x)}
$$

For $y=0$ eq. (17) gives

$$
u_{m} \frac{\partial \bar{q}}{\partial x}=-\frac{K \bar{q}^{2}}{\epsilon} \Rightarrow u_{m} \frac{\partial}{\partial x}\left(\frac{1}{\bar{q}}\right)=\frac{K}{\epsilon},
$$

because $K E_{x} \ll u_{m}$. For $\bar{q}(x)$, the partial derivative can be substituted by the total derivative. Hence, applying $u_{m} d / d x$ to $(29)$ we get

$$
u_{m} \frac{d}{d x}\left(\delta_{q} u_{m}\right)=\frac{K J}{2 \epsilon}
$$

Eqs. (31), (27) and (28) define the problem. In order to obtain a set of differential equations we have to express the integrals in (27) and (28) in terms of $u_{m}$ and $\delta_{l}$. This can be performed by imposing a velocity profile. If $\delta_{q}$ is clearly lower than $\delta_{l}$ the electric force remains confined into a very narrow region inside the plume, so that we can approximate the distribution $u(x, y)$ by a pseudo self-similar velocity profile $u(x, y)=u_{m}(x) f(\eta)$, with $\eta=y / \delta_{l}(x)$, $f(0)=1$ and $f^{\prime}(\eta) \leq 0 \forall \eta$. Therefore, we obtain three differential equations for $u_{m}(x), \delta_{l}(x)$ and $\delta_{q}(x)$,

$$
\begin{gathered}
u_{m} \frac{d}{d x}\left(u_{m}^{2} \delta_{l}\right)=\frac{1}{B_{1}} \frac{J E_{0}(x)}{\rho}, \\
B_{2} \frac{d}{d x}\left(u_{m}^{3} \delta_{l}\right)=-\nu B_{3} \frac{u_{m}^{2}}{\delta_{l}}+\frac{J E_{0}(x)}{\rho}, \\
u_{m} \frac{d}{d x}\left(u_{m} \delta_{q}\right)=\frac{J K}{2 \epsilon} .
\end{gathered}
$$

The constants are

$$
\begin{gathered}
B_{1}=\int_{-\infty}^{\infty} f^{2}(\eta) d \eta, \\
B_{2}=\int_{-\infty}^{\infty} \frac{1}{2} f^{3}(\eta) d \eta, \\
B_{3}=\int_{-\infty}^{\infty}\left(f^{\prime}\right)^{2}(\eta) d \eta .
\end{gathered}
$$

In order to estimate the values of $B_{1}, B_{2}$ and $B_{3}$, a velocity profile must be given. Choosing $f(\eta)=\exp (-\eta)$ as a plausible profile leads to $B_{1}=1, B_{2}=1 / 3, B_{3}=1$. Any other choice satisfying the boundary conditions gives similar values for the coefficients.

\section{Solutions for some particular dependences of the electric field}

If we assume that the electric field depends on $x$ as $E_{0}(x)=\bar{E}(x / d)^{m}$, we obtain

$$
u_{m}=C_{u}\left(\frac{J^{2} \bar{E}^{2}}{\rho^{2} \nu d^{2 m}}\right)^{1 / 5} x^{(1+2 m) / 5}
$$




$$
\begin{gathered}
\delta_{l}=C_{l}\left(\frac{\rho \nu^{3} d^{m}}{J \bar{E}}\right)^{1 / 5} x^{(2-m) / 5}, \\
\delta_{q}=C_{q} \frac{K}{\epsilon}\left(\frac{J \rho^{4} \nu^{2} d^{4 m}}{\bar{E}^{4}}\right)^{1 / 5} x^{(3-4 m) / 5}, \\
\frac{\delta_{q}}{\delta_{l}}=C_{q l} \frac{K}{\epsilon}\left(\frac{J^{2} \rho^{3} d^{3 m}}{\nu \bar{E}^{3}}\right)^{1 / 5} x^{(1-3 m) / 5} .
\end{gathered}
$$

The constants are

$$
\begin{gathered}
C_{u}=\left(\frac{B_{3}^{2}}{n_{1} B_{1}}\left(n_{1} B_{1}-n_{2} B_{2}\right)\right)^{1 / 5}, \\
C_{l}=\left(\frac{n_{1} B_{1} B_{3}^{3}}{\left(n_{1} B_{1}-n_{2} B_{2}\right)^{3}}\right)^{1 / 5}, \\
C_{q}=\left(\frac{B_{3}^{2}}{32 n_{1} n_{3}^{5} B_{1}}\left(n_{1} B_{1}-n_{2} B_{2}\right)\right)^{1 / 5}, \\
C_{q l}=\left(\frac{\left(n_{1} B_{1}-n_{2} B_{2}\right)^{4}}{32 n_{1}^{2} n_{3}^{5} B_{1}^{2} B_{3}}\right)^{1 / 5},
\end{gathered}
$$

with

$$
n_{1}=(4-3 m) / 5, n_{2}=1+m, n_{3}=(4-2 m) / 5 .
$$

In the case $m=0$ (uniform electric field), the expressions (34a) and (34b) are quite analogous to those obtained neglecting Coulomb repulsion. Therefore, for 2D EHD plumes, including Coulomb repulsion terms does not affect the hydrodynamic boundary layer. But now the charge boundary layer is characterized. Note that $\delta_{q}$ varies with $x$ in a way clearly different from $\delta_{l}$ and from the evolution predicted by Zhakin when only diffusion is retained.

Taking the same values used in IIC, with $m=0$, we obtain at $x=1 \mathrm{cm:} u_{m} \sim 8 \mathrm{~cm} \mathrm{~s}^{-1}, \delta_{1} \sim 2.2 \mathrm{~mm}, \delta_{\mathrm{q}} \sim$ $4 \mu \mathrm{m}, \delta_{\mathrm{q}} / \delta_{1} \sim 2 \times 10^{-3}$. Recalling (10), we see that the order of magnitude of $\delta_{q}$ is big enough for the effect of charge diffusion being negligible.

\section{B. Integral solutions for axisymmetric EHD plumes}

The previous analysis for 2D EHD plumes can be easily extended to axisymmetric plumes. Here, the flow is considered again to have a double boundary layer structure, with an inner charged core of radius $\delta_{q}(x)$ and an outer hydrodynamic layer of radius $\delta_{l}(x)$, with $\delta_{q}<\delta_{l}$. The total charge per unit length in a plane at constant $x$ is

$Q(x)=2 \pi \int_{0}^{+\infty} y q d y$, and the total current is $I=2 \pi \int_{0}^{+\infty} y q u d y \approx Q(x) u_{m}(x)$. From (1b) and (14), following the same steps than in $\S$ III A, we obtain the equations that describe the dynamics of axisymmetric EHD plumes

$$
\begin{gathered}
u_{m} \frac{d}{d x} \int_{0}^{\infty} y u^{2} d y=\frac{I E_{0}(x)}{2 \pi \rho} \\
\frac{d}{d x} \int_{0}^{\infty} \frac{1}{2} y u^{3} d y=-\nu \int_{0}^{\infty} y\left(\frac{\partial u}{\partial y}\right)^{2} d y+\frac{I E_{0}(x)}{2 \pi \rho} \\
u_{m} \frac{d}{d x}\left(u_{m} \delta_{q}^{2}\right)=\frac{I K}{\pi \epsilon} .
\end{gathered}
$$


Let us propose again a self-similar-like velocity profile $u(x, y)=u_{m}(x) f(\eta)$. By substituting in, (37a)-(37c) we obtain

$$
\begin{gathered}
u_{m} \frac{d}{d x}\left(u_{m}^{2} \delta_{l}^{2}\right)=\frac{1}{A_{1}} \frac{I E_{0}(x)}{2 \pi \rho}, \\
A_{2} \frac{d}{d x}\left(\delta_{l}^{2} u_{m}^{3}\right)=-A_{3} \nu u_{m}^{2}+\frac{I E_{0}(x)}{2 \pi \rho}, \\
u_{m} \frac{d}{d x}\left(\delta_{q}^{2} u_{m}\right)=\frac{K I}{\pi \epsilon} .
\end{gathered}
$$

The constants $A_{1}, A_{2}$ and $A_{3}$ are defined by

$$
\begin{gathered}
A_{1}=\int_{0}^{\infty} \eta f^{2}(\eta) d \eta \\
A_{2}=\int_{0}^{\infty} \frac{1}{2} \eta f^{3}(\eta) d \eta \\
A_{3}=\int_{0}^{\infty} \eta\left(f^{\prime}(\eta)\right)^{2} d \eta .
\end{gathered}
$$

Taking $f(\eta)=\exp (-\eta)$ leads to the numerical values $A_{1}=1 / 4, A_{2}=1 / 18, A_{3}=1 / 4$. For a given dependence of the longitudinal field on $x$, eqs. (38a)-(38c), along with their corresponding initial conditions, describe the evolution of the axisymmetric EHD plumes.

\section{Solutions for some particular dependences of the electric field}

By assuming the applied electric field to depend on the longitudinal coordinate through $E_{0}(x)=\bar{E}(x / d)^{m}$, the solutions to eqs. (38a)-(38c) are

$$
\begin{gathered}
u_{m}=\left(\frac{(1+m) A_{2}}{(1-m / 2) A_{1} A_{3}} \frac{I \bar{E}}{2 \pi \rho \nu}\right)^{1 / 2} x^{m / 2}, \\
\delta_{l}=\left(\frac{(1-m) A_{1} A_{3}^{3}}{(1+m)^{3} A_{2}^{2}} \frac{2 \pi \rho \nu^{3}}{I \bar{E}}\right)^{1 / 4} x^{1 / 2-m / 4}, \\
\delta_{q}=\left(\frac{(2-m) A_{1} A_{3}}{(1+m) A_{2}} \frac{\rho \nu K}{\epsilon \bar{E}}\right)^{1 / 2} x^{1 / 2-m / 2}, \\
\frac{\delta_{q}}{\delta_{l}}=\left(\frac{\left(1+m / 2-m^{2} / 2\right) A_{1}}{A_{3}} \frac{2 \rho K^{2} I}{\pi \nu \epsilon^{2} \bar{E}}\right)^{1 / 4} x^{-m / 4}
\end{gathered}
$$

As in 2D plumes, the expressions (40a) and (40b), with $m=0$, are similar to (24) obtained by neglecting Coulomb repulsion, and the evolution of the charged core is obtained. Note again that the behavior of $\delta_{q}$ differs from $\delta_{l}$ in the general case.

From experiments in transformer oil ${ }^{13}$ typical values of current and mean applied electric field are $I=10^{-7} \mathrm{~A}$ and $E_{0}=10^{6} \mathrm{~V} \mathrm{~m}^{-1}$, with $\epsilon_{r}=2$. Using an exponential profile to compute the constants $A_{i}$, we have the following estimated values at a distance $x=1 \mathrm{~cm}$ from the injector: $u_{\mathrm{m}} \sim 0.9 \mathrm{~m} \mathrm{~s}^{-1}, \delta_{\mathrm{l}} \sim 480 \mu \mathrm{m}, \delta_{\mathrm{q}} \sim 140 \mu \mathrm{m}, \delta_{\mathrm{q}} / \delta_{\mathrm{l}} \sim 0.3$. These estimates are not unconsistent with the approximations assumed in the derivation.

The flow field in axisymmetic plumes turns out to be much thinner than the $2 \mathrm{D}$ ones. On the other hand, consistently with the basic picture, $\delta_{q}$ is clearly smaller than $\delta_{l}$, but not much smaller. 


\section{Numerical computations}

Eqs. (32) or (38) are readily integrable numerically. But, as it is pointed out in $\S$ III A, the dependence on $x$ of the longitudinal electric field, $E_{0}(x)$, and the initial conditions for $u_{m}, \delta_{l}$ and $\delta_{q}$ must be given. The determination of the initial conditions implies to solve the whole EHD problem close to the needle tip, and, as we mentioned in the Introduction, this is a very difficult task. However, as in this paper we are interested in determining the behavior of EHD plumes far from both electrodes, we have solved numerically the problem with an heuristic imposed electric field and several sets of initial conditions for axisymmetric plumes, in order to study the asymptotic behavior. All the calculations have been made using a fourth-order Runge-Kutta algorithm ${ }^{21}$.

The real dependence of the longitudinal electric field on $x$ is rather complicated. Close to the point electrode its value is always around $E_{s}$, and it decreases rapidly away from the injector, taking values of the order of $V / d$ in the volume. Previous works on wire-cylinder geometry ${ }^{22,23}$ have shown that the behavior of the electric field close to the injector does not differ too much from the harmonic field and, in the inter-electrodes volume, remains nearly constant. Following this, we have imposed the following profile of the longitudinal electric field,

$$
E_{0}(x)=\left\{\begin{array}{cl}
E_{s} \frac{1-\left(1-r_{0} / d\right)^{2}}{1-(1-x / d)^{2}} & r_{0}<x \leq \bar{x}, \\
\bar{E}=E_{s} \frac{1-\left(1-r_{0} / d\right)^{2}}{1-(1-\bar{x} / d)^{2}} & \bar{x}<x<d .
\end{array}\right.
$$

The value $\bar{x}$ is determined from the boundary conditions on the electric potential. Taking $I=1.12 \times 10^{-7} \mathrm{~A}, \mathrm{~V}=$ $20 \mathrm{kV}, \mathrm{d}=2 \mathrm{~cm}, \rho=850 \mathrm{~g} \mathrm{~cm}^{-3}, \nu=2.26 \times 10^{-5} \mathrm{~m}^{2} \mathrm{~s}^{-1}$ and $K=10^{-9} \mathrm{~m}^{2} \mathrm{~V}^{-1} \mathrm{~s}^{-1}$ (these values are obtained from experiments with transformer oil ${ }^{24}$ ) it is $\bar{x}=0.300 d=6.0 \mathrm{~mm}$ and $\bar{E}=3.92 \times 10^{5} \mathrm{~V} \mathrm{~m}^{-1}$. Figure 3 shows this longitudinal electric field. Figures 4 and 5 show the several computations for different initial values of $\lambda=\delta_{l} / \delta_{q}$. In all cases the initial conditions for the velocity and $\delta_{q}$ are $u_{m}(0)=5 \mathrm{~m} \mathrm{~s}^{-1}$ and $\delta_{q}(0)=r_{0}=5 \mu \mathrm{m}$.

We observe that the velocity grows very quickly near the injector, because of the high value of the electric field there. The maximum of the velocity depends greatly on the initial value of the hydrodynamic radius. High values of $\delta_{l}(0)$ imply that a large portion of the liquid is put into motion and, consequently, the acceleration is smaller. When the electric field decreases, the acceleration also decreases, until the viscous stresses overcome the driving force, giving a negative acceleration.

On the other hand, the radius $\delta_{q}$ first decreases due to the constriction effect produced by the acceleration of the liquid. When this acceleration decreases the charged core grows and in the region of uniform electric field $\delta_{q}$ exhibits the asymptotic behavior given by (40c). A similar behavior holds for $\delta_{l}$, but the initial decreasing is only important for high values of $\delta_{l}(0)$. As a consequence, the ratio $\delta_{l} / \delta_{q}$, which varies quickly in the high-field region for moderate enough values of $\delta_{l}(0)$, tends to a constant value $(\lambda \approx 6)$ in the uniform field region as given by (40d).

Note that, in the uniform field region, all the variables follow the asymptotic behavior given by (40), independently of the initial conditions.

\section{DISCUSSION AND CONCLUSIONS}

The dynamics and the charge conduction mechanisms in EHD plumes have been analyzed, for both 2D and axisymmetric geometries, in the region far from both electrodes (the asymptotic region). The relative importance in the transport of charge of diffusion, drift by the electric field and convection by the fluid has been considered. In both axisymmetric and 2D plumes, the longitudinal diffusion of charge is neglected compared to the drift. In axisymmetric plumes, the transverse diffusion of charge is negligible compared to transverse drift for any value of the radius of the inner charged core, for the known experimental values of current intensity and ionic mobility. In the $2 \mathrm{D}$ case, diffusion is negligible only if the width of the charged layer is greater than a certain value $\left(\delta_{q}^{l i m} \sim 1 \mu \mathrm{m}\right.$ in usual experimental conditions). In this paper, charge diffusion has been neglected in studying the dynamics of EHD plumes.

The longitudinal drift is always negligible compared to the convection of the fluid. But the transverse drift, due to the electric field created by the space charge distribution (the Coulomb repulsion), can be of the same order of magnitude than transverse convection. The quotient, $\Omega(x)=t(x) / \tau_{r}$, where $t$ is the time during which Coulomb repulsion acts, and $\tau_{r}=\epsilon / K q_{i}$, the typical evolution time of the density of charge, determines when the Coulomb repulsion must be taken into account. The initially injected density of charge, $q_{i}$, has been estimated from experimental data for both geometries. Only in 2D plumes the Coulomb repulsion can be neglected for low enough values of the current. But in general it has to be taken into account.

When Coulomb repulsion is negligible and $\delta_{q} \rightarrow 0$, self-similar solutions have been found elsewhere ${ }^{5,6}$. But, if Coulomb repulsion must be considered, as it is usually the case, this analysis does not apply anymore. Imposing 
conservation of momentum, energy and charge, averaged over the transverse section of the plume, a set of three integrodifferential equations have been obtained. Assuming a pseudo self-similar velocity profile $u(x, y)=u_{m}(x) f(\eta)$, with $\eta=y / \delta_{l}(x)$, they can be reduced to a set of three differential equations, which do not depend on the profile $f(\eta)$, except for the numerical values of three constants. These equations, given the longitudinal electric field, $E_{0}(x)$ along with the initial conditions, describe the evolution of the EHD plumes.

In the case of a power law dependence on $x$ of the electric field, solutions with a similar dependence can be found. In this way, the evolution of the charged core is obtained, which was not given by the previous models. The numerical estimation, in usual experimental conditions, gives $\delta_{q} / \delta_{l} \sim 2 \times 10^{-3}$ for $2 \mathrm{D}$ plumes and $\delta_{q} / \delta_{l} \sim 0.3$ for axisymmetric ones. This estimation justify the assumption of charge diffusion to be negligible. The charged core turns out to be clearly smaller than the hydrodynamic layer, consistently with the assumptions of the model, but in the axisymmetric case, $\delta_{q}$ is not much smaller than $\delta_{l}$.

The evolution of $u_{m}(x)$ and $\delta_{l}(x)$ turns out to be analogous to the behavior obtained when neglecting Coulomb repulsion, even in the axisymmetric case. As far as the electric force is confined into the hydrodynamic layer, the asymptotic behavior of $\delta_{l}(x)$ must be controlled by the diffusion of vorticity, regardless of the detailed structure of the charged core, and in accordance with the basic scales stated in II C.

The differential equations have been numerically integrated for the axisymmetric case using an heuristic approximation of the longitudinal electric field. We have considered an harmonic-like field near the injector and an uniform electric field in the region between electrodes. The asymptotic dependences are reobtained, independently of the initial conditions.

\section{ACKNOWLEDGMENTS}

This work was carried out with financial support from DGiCYT (Spanish Government Agency) PB96-1375. We are indebted to the referees for their comments, that have improved this paper. 
1. E. A. Castellanos, Electrohydrodynamics (Springer, 1998).

2. Y. Jaluria, Natural Convection Heat and Mass Transfer (Pergamon, New York, 1980).

3. B. Gebhart, Y. Jaluria, R. Mahajan, and B. Sammakia, Buoyancy-Induced Flows and Transport (Hemisphere Publishing Corporation, New York, 1988).

4. A. Zhakin, "Electroconvective jets in liquid dielectrics," Mekhanica Zhigkosty i Gaza. Isvestia Academy Science USSR 6, 13-19 (1984).

5. A. T. Pérez, P. A. Vázquez, and A. Castellanos, "Dynamics and linear stability of charged jets in dielectric liquids," IEEE Transactions on Industry Applications 31, 761-768 (1995).

6. P. A. Vázquez, A. T. Pérez, and A. Castellanos, "Thermal and electrohydrodynamical plumes. A comparative study," Physic of Fluids 8, 2091-2096 (1996).

7. D. B. Spalding and R. G. Crudace, "Theory of the steady laminar buoyant flow above a line heat source in a fluid of large Prandtl number and temperature-dependent viscosity," International Journal of Heat and Mass Transfer 3, 55-59 (1961).

8. H. K. Kuiken and Z. Rotem, "Asymptotic solution for plume at very large and small Prandtl numbers," Journal of Fluid Mechanics 45, 585-599 (1971).

9. F. Higuera and P. Vázquez, "Coulomb-driven flow of a dielectric liquid subject to charge injection by a sharp electrode," Physics of Fluids 11, 2434-2436 (1999).

10. T. Takashima, R. Hanaoka, R. Ishibashi, and A. Ohtsubo, "I-V Characteristics and liquid motion in needle-to-plane and razor blade-to-plane configurations in transformer oil and liquid nitrogen," IEEE Transactions on Electrical Insulation 23, 645-657 (1988).

11. F. M. J. McCluskey and A. T. Pérez, "The electrohydrodynamic plume between a line source of ions and a flat plate," IEEE Trans. on electrical insulation 27, 334-341 (1992).

12. B. Malraison, P. Atten, and A. Pérez, "Panaches chargés résultant de l'injection d'ions dans un liquide isolant par une lame ou une pointe placée en face d'un plan," Journal de Physique III 4, 75-85 (1994).

13. P. Atten, B. Malraison, and M. Zahn, "Electrohydrodynamic plumes in point-plane geometry," IEEE Transactions on Dielectric and Electric Insulations pp. 710-718 (1997).

14. G. K. Batchelor, An Introduction to Fluid Dynamics (Cambridge University Press, 1985).

15. E. Hopfinger, J. Lacroix, and P. Atten, "Electro-convection in a dielectric liquid layer subjected to unipolar injection," Journal of Fluid Mechanics 69, pp. 539-563 (1975).

16. E. Durand, Electrostatique (Masson, 1966), Vol. 2.

17. R. Sigmond, "Simple approximate treatment of unipolar space-charge-dominated coronas: The Warburg law and the saturation current," Journal of Applied Physics 53, 891-898 (1982).

18. R. Coelho and J. Debeau, "Properties of the tip-plane configuration," J. Phys. D: Appl. Phys. 4, 1266-1280 (1971).

19. N. Bonifaci and A. Denat, Conf. Rec. ICDL96 (1996), pp. 15-19.

20. A. Denat, J. P. Gosse, and B. Gosse, "Electrical conduction of purified cyclohexane in a divergent electric field," IEEE Transactions on Electrical Insulation 23, 545-554 (1988).

21. W. Press, B. Flannery, S. Teulosky, and W. Vetterling, Numerical Recipes in C (Cambridge University Press, 1990).

22. P. Atten and M. Haidara, "IEEE Electrical Conduction and EHD Motion of Dielectric Liquids in a Knife-Plane Electrode Assembly," Transactions on Electrical Insulation 20, 187-198 (1985).

23. F. Pontiga and A. Castellanos, "A dissociation-injection model for non-polar liquid conduction and wire-cylinder geometry," IEEE Transactions on Dielectrics and Electrical Insulation 4, 224-237 (1997).

24. P. A. Vázquez, Ph.D. thesis, Universidad de Sevilla, 1998. 


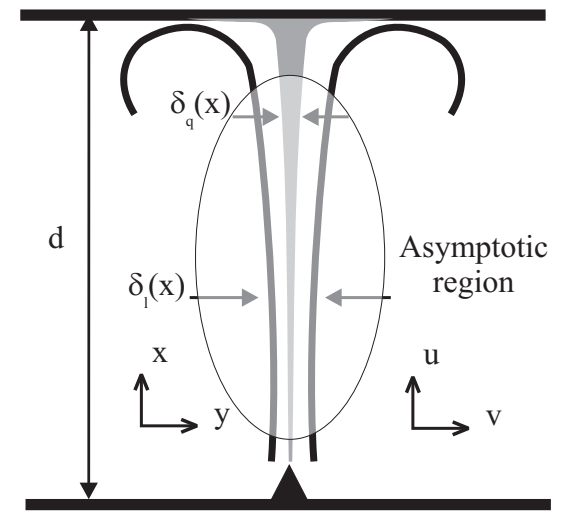

FIG. 1. Overall structure of electrohydrodynamic plumes. The solutions are found in the asymptotic region, far from both electrodes.

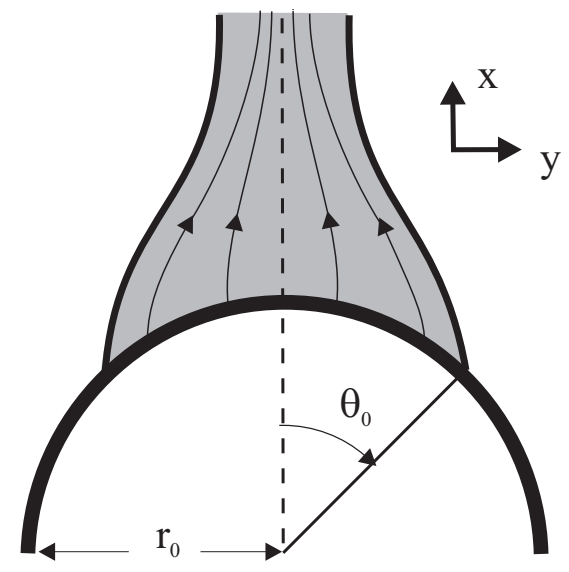

FIG. 2. Evolution of ions in the proximity of the injector. The shaded region is the charged zone.

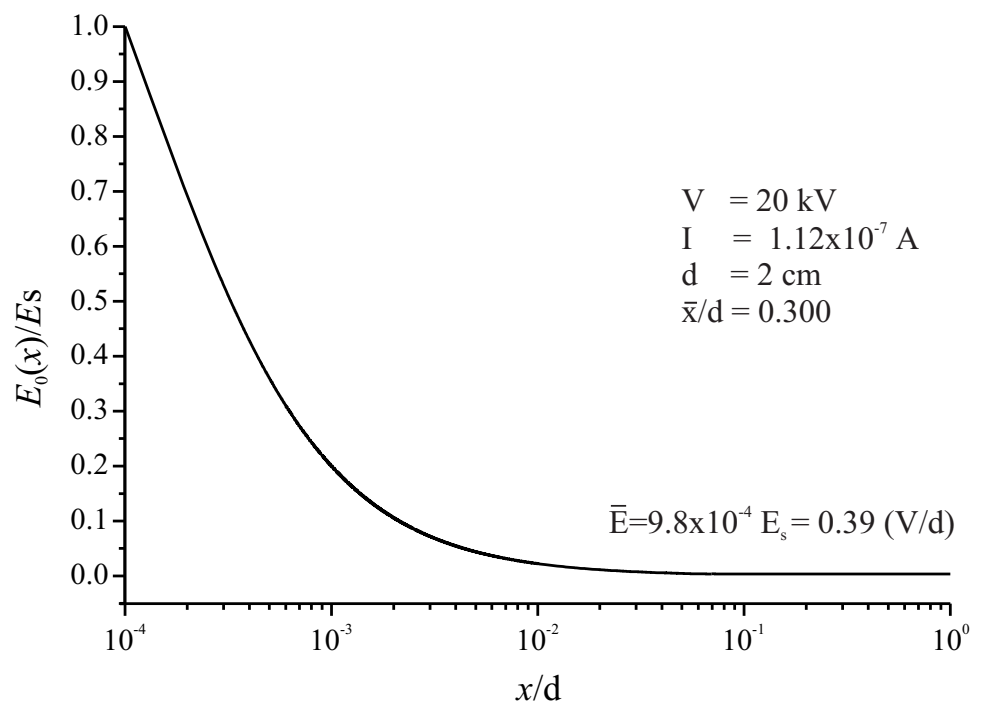

FIG. 3. Imposed longitudinal electric field. Near the injector the field is taken to have the harmonic dependence on $x$. Beyond a certain value $\bar{x}$, the field is taken to be constant. The value $\bar{x}$ is chosen to give the applied potential difference. The electric field is normalized with the threshold field $E_{s}=E_{0}(0)$. 

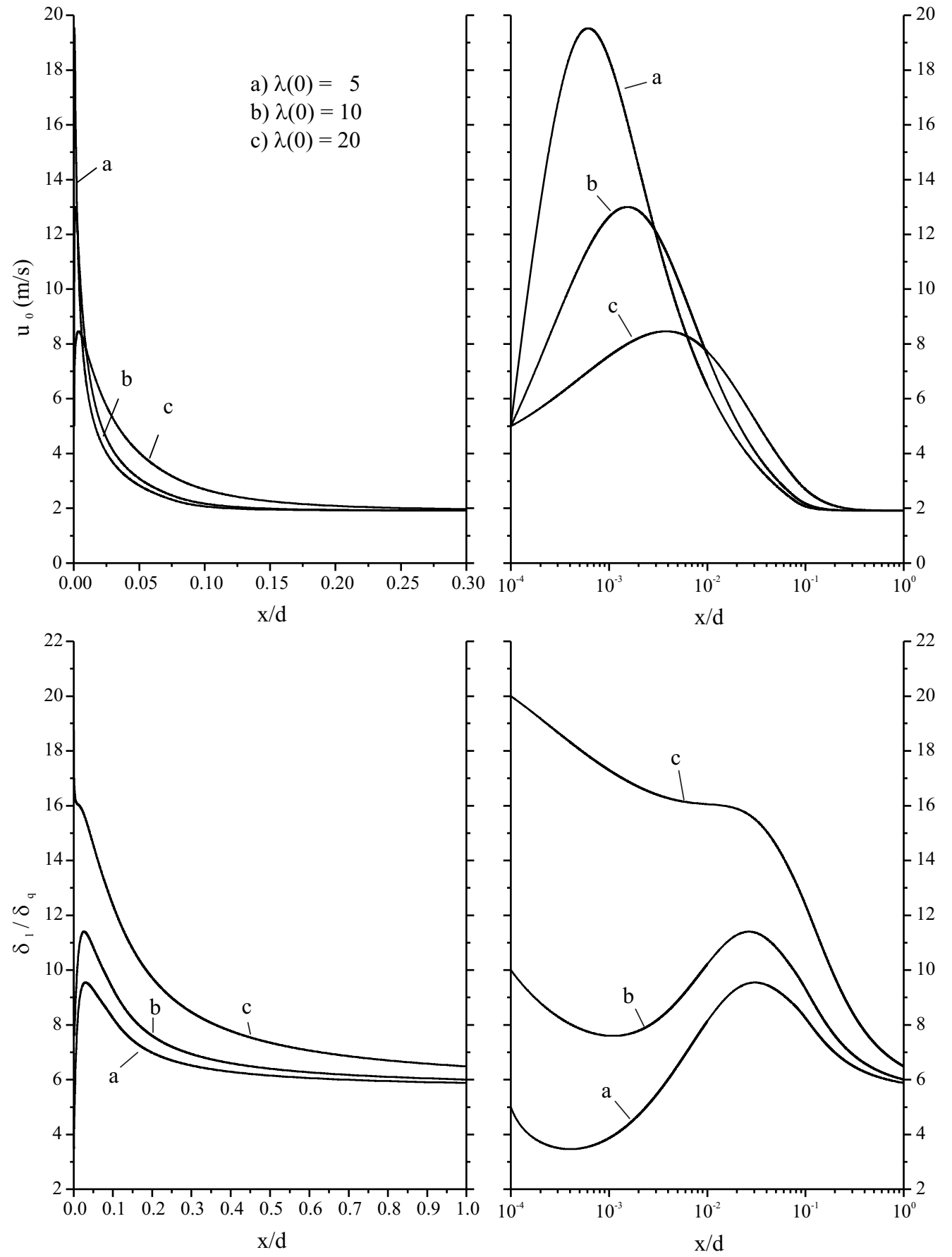

FIG. 4. Evolution of the longitudinal velocity and the ratio, $\lambda=\delta_{l} / \delta_{q}$, for different initial values of $\lambda$, with an imposed electric field. Linear and logarithmic scales in $x / d$ are used. 

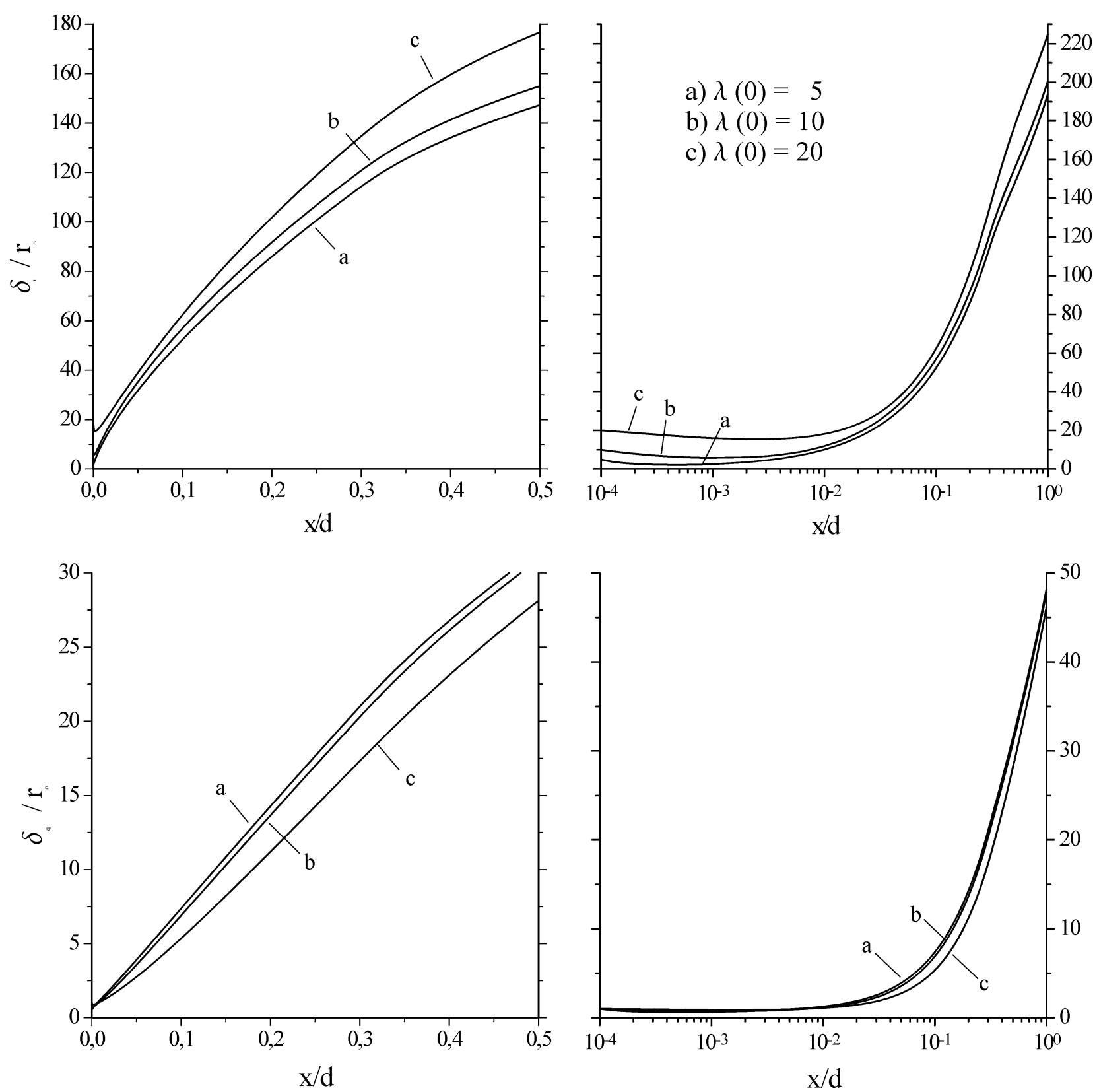

FIG. 5. Evolution of the thickness of the hydrodynamic layer, $\delta_{l}$ and the charged layer, $\delta_{q}$, for different initial values of $\lambda$, with an imposed electric field. Linear and logarithmic scales in $x / d$ are used. 